\title{
Understanding the Optics to Aid Microscopy Image Segmentation
}

\author{
Zhaozheng Yin ${ }^{1}$, Kang $\mathrm{Li}^{2}$, Takeo Kanade ${ }^{1}$, and Mei Chen ${ }^{3}$ \\ 1 Carnegie Mellon University \\ 2 Microsoft Corporation \\ 3 Intel Labs Pittsburgh
}

\begin{abstract}
Image segmentation is essential for many automated microscopy image analysis systems. Rather than treating microscopy images as general natural images and rushing into the image processing warehouse for solutions, we propose to study a microscope's optical properties to model its image formation process first using phase contrast microscopy as an exemplar. It turns out that the phase contrast imaging system can be relatively well explained by a linear imaging model. Using this model, we formulate a quadratic optimization function with sparseness and smoothness regularizations to restore the "authentic" phase contrast images that directly correspond to specimen's optical path length without phase contrast artifacts such as halo and shade-off. With artifacts removed, high quality segmentation can be achieved by simply thresholding the restored images. The imaging model and restoration method are quantitatively evaluated on two sequences with thousands of cells captured over several days.
\end{abstract}

\section{Introduction}

Long-term monitoring of living specimen's movement and behavior has a wide range of applications in biological discovery. Since transparent specimens such as living cells generally lack sufficient contrast to be observed by common light microscopes, the phase contrast imaging technique was invented to convert the minute light phase variation caused by specimen into changes in light amplitude that can be observed by naked eyes or cameras [15. Due to the optical principle and some imperfections of the conversion process, phase contrast images contain artifacts such as the halo surrounding the specimen and shade-off (Fig. 3(b), the intensity profile of a large specimen gradually increases from the edges to the center, and even approaches the intensity of the surrounding medium). Over time, biologists have learned how to overcome or even exploit those artifacts for interpreting images. When computer-based microscopy image analysis began to relieve humans from tedious manual labelling [581214, it is unsurprising that those artifacts cause the major difficulty in automated image processing. In particular, they hinder the process of segmenting images into cells and background, which is the most critical step in almost all cell analysis and tracking systems. 


\subsection{Previous Work}

In microscopy image segmentation, specimen pixels are segmented from background pixels and then grouped into objects. In the past decades, many image segmentation methods have been invented. For example, thresholding on local intensity value and variation has a long history on cell image segmentation ( 13 and references therein). Morphological operations on the gradient or intensity images are widely applied to segment specimen pixels [58]. Using the artifact of microscopy images, Laplacian of Gaussian filter is also used to extract object blob and contour [12. After obtaining binary masks indicating whether each pixel is a specimen or background pixel, connected component labelling or marker-controlled watershed algorithms are often performed to group specimen pixels into specimen objects [5]14.

\subsection{Our Proposal}

The previous image segmentation methods do not consider the microscopy image formation process and treat them in the same way as general natural images. However, there are apparent differences between natural images and phase contrast microscopy images, such as halo and shade-off. Because of these artifacts, the observed image intensity does not exactly correspond to specimen's optical path length (product of refractive index and thickness). Recently, Li and Kanade 9] proposed an algebraic framework for preconditioning microscopy images captured by differential interference contrast (DIC) microscopes. This inspired us to think about whether understanding the phase contrast optics at an early stage will help segment phase contrast images. In fact, we found that those artifacts are not caused by random processes, instead they can be relatively well modeled by the optical properties of the image formation process in the phase contrast microscope imaging system. In this paper, we derive a linear imaging model for phase contrast microscopy and formulate a quadratic optimization function to restore the "authentic" phase contrast images without halo or shade-off effects. With artifacts removed, high quality segmentation can be achieved by simply thresholding the restored images.

\section{Imaging Model of Phase Contrast Microscopy}

Phase contrast microscope uses a conjugate pair of condenser annulus and phase plate as shown in Fig. 1(c), where the specialized annulus is placed at the front focal plane of the condenser while the phase plate is at the rear focal plane of the objective lens. Wavefronts illuminate the specimen and divide into two components: one component passes through and around the specimen without deviation (commonly referred to as the $\mathrm{S}$ wave); the other component is diffracted, attenuated and retarded because of specimen ( $\mathrm{D}$ wave). A typical phase retardation caused by living cells in tissue culture is a quarter wave length [10]. The two waves enter the objective lens and combine through interference to produce the particle wave ( $\mathrm{P}$ wave). 


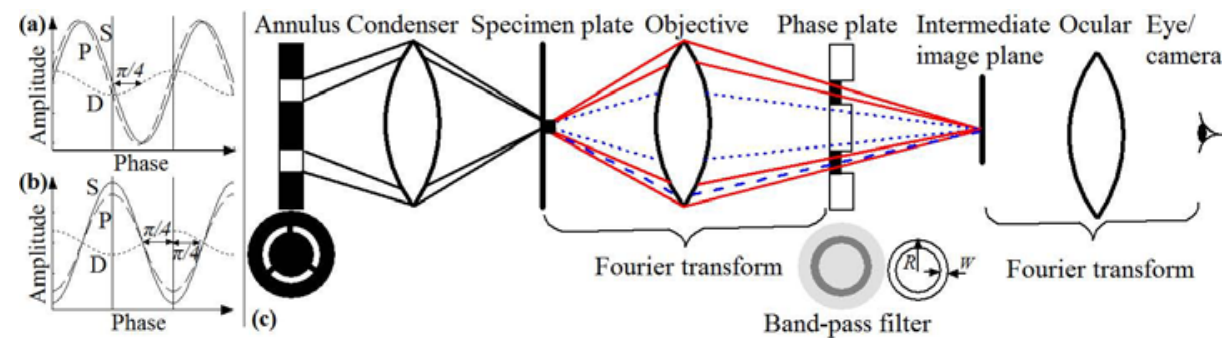

Fig. 1. Wave interaction and phase contrast microscope optics. (a) Without phase contrast, the amplitude difference between $\mathrm{P}$ (dash) and S (solid) waves is small; (b) With phase contrast technique, the difference is large enough to be observed. (c) The phase plate separates the surround (solid) and diffracted (dot) lights, speeds up (i.e. advances the phase by $1 / 4$ wavelength) and attenuates the surround light. A small portion of diffracted light (dash) leaking into the phase ring causes the halo and shadeoff artifacts.

Observing the specimen in the microscopy image depends on the intensity difference between specimen and its surrounding background, i.e. the amplitude difference between particle $(\mathrm{P})$ and surround (S) waves. Without any phase contrast technique, the $\mathrm{P}$ and $\mathrm{S}$ waves have nearly the same wave amplitudes as shown in Fig. 1(a), thus the specimen remains transparent and appears invisible. However, in phase contrast microscope (Fig. 1(c)), the phase plate has an etched ring with reduced thickness to advance the surround wave by a quarter wavelength. The phase ring also has a partially absorbing metallic film to attenuate the surround wave such that the final contrast between $\mathrm{P}$ and $\mathrm{S}$ waves are easy to be perceived. The diffracted wave spreads over the phase plate. Most of it passes through the phase plate without changes, and it interferes with the surround wave to form the particle wave as shown in Fig. 1)(b) where the amplitude difference between $\mathrm{P}$ and $\mathrm{S}$ waves is now observable and the specimen will appear as dark on a bright background. Unfortunately, some of the diffracted wave will "leak" into the phase ring, which cause the halo and shade-off artifacts.

After illuminating waves pass through the specimen plate, the unaltered surround wave $l_{S}(\mathbf{x})$ and the diffracted wave $l_{D}(\mathbf{x})$ (attenuated and retarded) are

$$
\begin{aligned}
& l_{S}(\mathbf{x})=A e^{i 0} \\
& l_{D}(\mathbf{x})=\zeta_{c} A e^{-i f(\mathbf{x})}
\end{aligned}
$$

where $i^{2}=-1, \mathbf{x}=\left\{\left(\mathbf{x}_{j}^{r}, \mathbf{x}_{j}^{c}\right), j=1, \cdots, J\right\}$ represent $2 \mathrm{D}$ locations of $J$ image pixels, $A$ is the illuminating wave's amplitude, $\zeta_{c}$ and $f(\mathbf{x})$ represent the amplitude attenuation and phase shift caused by the specimen. Our goal is to restore $f(\mathbf{x})$, the "authentic" phase contrast image without artifacts. A thin lens with a large aperture essentially performs a spatial Fourier transform $(\mathcal{F})$ on the waves from its front focal plane to its rear focal plane [3], thus the waves in front of the phase plate are $L_{S}(\mathbf{w})=\mathcal{F}\left(l_{S}(\mathbf{x})\right)$ and $L_{D}(\mathbf{w})=\mathcal{F}\left(l_{D}(\mathbf{x})\right)$. 
The phase plate functions as a band-pass filter. For the non-diffracted surround wave, the positive phase ring attenuates the wave amplitude and advances its phase by a quarter wave length $(\pi / 2)$, thus the corresponding transmittance function is

$$
T_{S}(\mathbf{w})=\zeta_{p} e^{i \frac{\pi}{2}}=i \zeta_{p}
$$

where $\zeta_{p}$ represents the amplitude attenuation by a phase ring with outer radius $R$ and width $W$ ( $R$ and $W$ are provided by microscope manufactures). The diffracted wave spreads over the phase plate with a small portion leaking into the ring. The corresponding transmittance function is

$$
T_{D}(\mathbf{w})=1+\left(i \zeta_{p}-1\right)\left[\operatorname{cyl}\left(\frac{w_{r}}{R}\right)-\operatorname{cyl}\left(\frac{w_{r}}{R-W}\right)\right]
$$

where $w_{r}=\sqrt{w_{u}^{2}+w_{v}^{2}}$ is the radial frequency and cyl $(\cdot)$ is a $2 \mathrm{D}$ cylinder (or circular) function: $\operatorname{cyl}(t)=1$, if $0 \leq \mathrm{t} \leq 1$; 0 , otherwise. After band-pass filtering, we have the waves immediately after the phase plate as $\tilde{L}_{S}(\mathbf{w})=L_{S}(\mathbf{w}) T_{S}(\mathbf{w})$ and $\tilde{L}_{D}(\mathbf{w})=L_{D}(\mathbf{w}) T_{D}(\mathbf{w})$.

The ocular lens perform another consecutive Fourier transform. Mathematically, the forward and inverse Fourier transforms are identical except for a minus sign. Hence, we have the waves after the ocular lens as $\tilde{l}_{S}(\mathbf{x})=l_{S}(\mathbf{x}) * t_{S}(\mathbf{x})$ and $\tilde{l}_{D}(\mathbf{x})=l_{D}(\mathbf{x}) * t_{D}(\mathbf{x})$, where $*$ denotes the convolution operator. $t_{S}(\cdot)$ and $t_{D}(\cdot)$ are the inverse Fourier transform of $T_{S}(\cdot)$ and $T_{D}(\cdot)$, respectively

$$
\begin{aligned}
t_{S}(\mathbf{x}) & =i \zeta_{p} \delta(\mathbf{x}) \\
t_{D}(\mathbf{x}) & =\delta(\mathbf{x})+\left(i \zeta_{p}-1\right) \operatorname{airy}(r)
\end{aligned}
$$

where $\delta()$ is a Dirac delta function, $r=\sqrt{u^{2}+v^{2}}$ is the radial distance and $\operatorname{airy}(r)$ is an obscured Airy pattern [1]

$$
\operatorname{airy}(r)=R \frac{J_{1}(2 \pi R r)}{r}-(R-W) \frac{J_{1}(2 \pi(R-W) r)}{r}
$$

and $J_{1}(\cdot)$ is the first order Bessel function of the first kind. Fig. 2 shows a sample of obscured Airy pattern where a bright region in the center is surrounded by a series of concentric bright/dark rings.

Substituting $l_{S}, t_{S}, l_{D}, t_{D}$ into $\tilde{l}_{S}$ and $\tilde{l}_{D}(\mathbf{x})$, we get

$$
\begin{aligned}
& \tilde{l}_{S}(\mathbf{x})=i \zeta_{p} A \\
& \tilde{l}_{D}(\mathbf{x})=\zeta_{c} A e^{-i f(\mathbf{x})}+\left(i \zeta_{p}-1\right) \zeta_{c} A e^{-i f(\mathbf{x})} * \operatorname{airy}(r)
\end{aligned}
$$

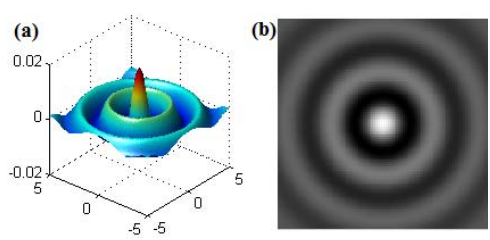

Fig. 2. An obscured Airy pattern. (a) 3D surface view; (b) 2D view. 
The first term in Eq9 is the primary component of the diffracted wave that destructively interferes with the non-diffracted surround wave and generates the contrast for human observation. The second term in Eq9 comes from the diffracted wave leaking into the phase ring which causes the halo and shade-off artifacts. The intensity of the final observed image is computed as

$$
\begin{aligned}
g(\mathbf{x}) & =\left|\tilde{l}_{S}(\mathbf{x})-\tilde{l}_{D}(\mathbf{x})\right|^{2} \\
& \propto(\delta(r)-\operatorname{airy}(r)) * f(\mathbf{x})+C
\end{aligned}
$$

where $C$ is a constant. The convolution kernel in Eq11 represents the point spread function (PSF) of the phase contrast microscope

$$
\operatorname{PSF}(u, v)=\delta(u, v)-\operatorname{airy}\left(\sqrt{u^{2}+v^{2}}\right)
$$

which is a linear operator.

Now we can define the linear imaging model between $\mathbf{g}$ (observed image) and f (artifact-free phase contrast image to be restored) as

$$
\mathrm{g} \approx \mathbf{H f}+C
$$

In practice, we discretize the PSF kernel as a $(2 \mathrm{M}+1) \times(2 \mathrm{M}+1)$ matrix (e.g. $M=5$ ) and the $\mathbf{H}$ matrix is defined by

$$
(\mathbf{H f})_{j}=\sum_{u=1}^{2 M+1} \sum_{v=1}^{2 M+1} \operatorname{PSF}(u, v) \mathbf{f}\left(\mathbf{x}_{j}^{r}+u-M, \mathbf{x}_{j}^{c}+v-M\right)
$$

\section{Restoring Artifact-Free Phase Contrast Image}

Now that the phase-contrast microscopy imaging model is established as in Eq 13, we develop procedures in this section to restore $\mathbf{f}$ from $\mathbf{g}$. The first step is to remove the background $C$ from $\mathbf{g}$ by flat-field correction 10 , after which we re-define $\mathbf{g}$ as the corrected image, i.e. $\mathbf{g} \leftarrow \mathbf{g}-C$. The second and major step is to solve $\mathbf{f}$ from $\mathbf{g} \approx \mathbf{H f}$. An attempt to solve this by simply inversing $\mathbf{H}$ is known to be highly noise-prone. Instead, we formulate the following constrained quadratic function to restore $\mathbf{f}$

$$
\mathbf{O}(\mathbf{f})=\|\mathbf{H} \mathbf{f}-\mathbf{g}\|_{2}^{2}+\omega_{s} \mathbf{f}^{T} \mathbf{L f}+\omega_{r}\|\mathbf{\Lambda} \mathbf{f}\|_{p}^{p}
$$

where $\mathbf{L}$ and $\boldsymbol{\Lambda}$ are Laplacian matrix and diagonal matrix defining the local smoothness and sparseness with corresponding weights $\omega_{s}$ and $\omega_{r}$, and $\|\cdot\|_{p}$ denotes the $l_{p}$-norm. A similarity-based Laplacian matrix is defined as $\mathbf{L}=$ $\mathbf{D}-\mathbf{W}$ where $\mathbf{W}$ is a symmetric matrix whose off-diagonal elements are defined as $\mathbf{W}(i, j)=e^{-\left(\mathbf{g}_{i}-\mathbf{g}_{j}\right)^{2} / \sigma^{2}}$. Typically, $\sigma^{2}$ is chosen by hand [4] or computed as the mean of all $\left(\mathbf{g}_{i}-\mathbf{g}_{j}\right)^{2}$ 's. $\mathbf{D}$ is a diagonal degree matrix where $\mathbf{D}(i, i)=$ $\sum_{j} \mathbf{W}(i, j)$. The image matting Laplacian matrix proposed by Levin et al. [7] can also be applied here.

When using $l_{2}$ sparseness regularization, $\mathbf{O}(\mathbf{f})$ can be expressed as

$$
\mathbf{O}(\mathbf{f})=\mathbf{f}^{T} \mathbf{Q} \mathbf{f}-2 \mathbf{b}^{T} \mathbf{f}+\mathbf{g}^{T} \mathbf{g}
$$

where

$$
\begin{aligned}
\mathbf{Q} & =\mathbf{H}^{T} \mathbf{H}+\omega_{s} \mathbf{L}+\omega_{r} \boldsymbol{\Lambda}^{T} \boldsymbol{\Lambda} \\
\mathbf{b} & =\mathbf{H}^{T} \mathbf{g}
\end{aligned}
$$


A closed-form solution of $\mathbf{f}$ can be achieved by solving a sparse linear system of equations $\mathbf{Q f}=\mathbf{b}$. Available solvers include conjugate gradient method or Matlab backslash operator (used in 47]). When using $l_{1}$ regularization, there is no closed-form solution and only numerical approximation is available. We combine non-negative multiplicative update [11] and re-weighting techniques [2] to solve the $l_{1}$ regularized objective function [9].

\section{Experiments}

Fig. 3 shows several restored samples by solving the $l_{1}$-regularized quadratic problem based on the derived imaging model. We will discuss the effects of different regularization terms and corresponding solvers in Section 4.1. In Fig. 3 . it appears that the restored artifact-free images are easier to be segmented because the cells are represented by bright (positive-valued) pixels set on a uniformly black (zero-valued) background. To see if this is the case, we have done quantitative evaluation of segmentation by using restored images in Section 4.2 .

\subsection{Smoothness and $l_{p}$ Sparseness Regularizations}

When using $l_{2}$ sparseness regularization in Eq,15, we can restore a closed-form solution $\mathbf{f}$ (Fig. $4(\mathrm{a})$ ) from $\mathbf{g}$ (Fig[3(d)). This closed-form solution has both positive and negative values corresponding to cell pixels (Fig. 4(b)) and halo/mitotic cell pixels (Fig. 4(c)) respectively. Comparing Fig. 33(i) with Fig. 4(b), we find that $l_{2}$ regularization does not enhance sparseness as well as $l_{1}$ regularization does. This phenomenon is also discussed in [6. When there is no sparse constraint, the restored image includes many background pixels (Fig. 4(d)). When there is no smoothness constraint, gaps between object parts such as cell nuclei and membrane may appear (Fig. 4(e)). Without smooth or sparse constraints, the directly solved $\mathbf{f}$ by inversing $\mathbf{H}$ is quite noisy (Fig. $4(\mathrm{f})$ ).

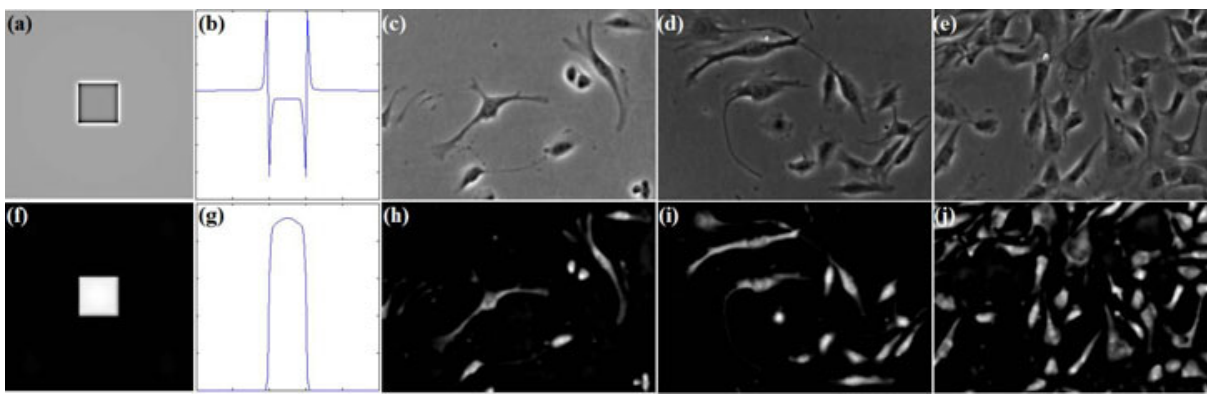

Fig. 3. Restore artifact-free phase contrast images. (a) synthesized phase contrast image; (b) intensity profile of the central row of (a) (or one may think of it as a 1D phase contrast image); (c)-(e) real phase contrast microscopy images with increasing cell densities; (f)-(j) restored artifact-free images corresponding to (a)-(e). 

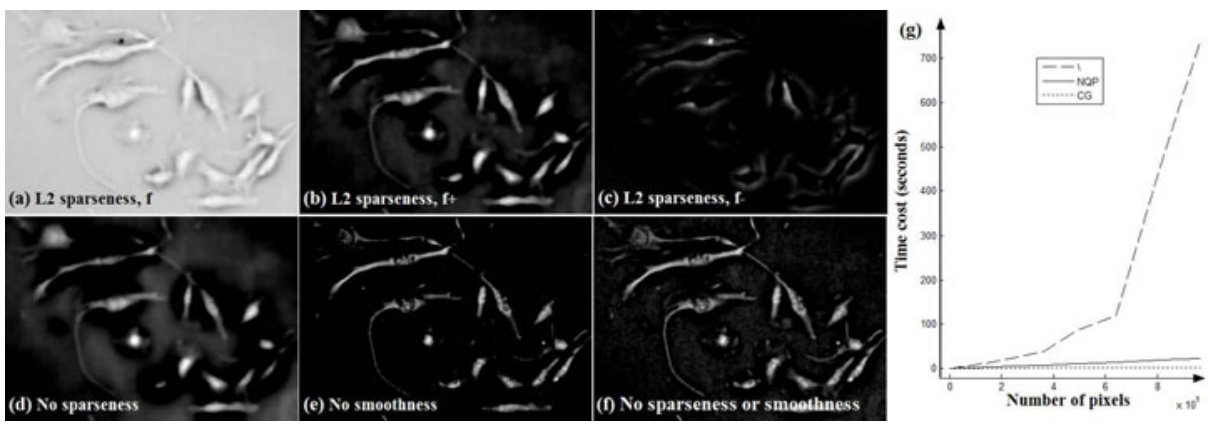

Fig. 4. Sparseness and smoothness. (a) restored image by applying conjugate gradient method $(\mathrm{CG})$ to solve $O(\mathbf{f})$ with $l_{2}$ sparseness regularization; (b) and(c) the positive and negative part of $(\mathrm{a})$; (d) restored image without sparseness; (e) restored image without smoothness; (f) restored image without sparseness or smoothness; (g) computational cost comparison.

In terms of computational cost (Fig. 4(g)), the nonnegative quadratic programming solver (NQP) for $l_{1}$-regularized $O(\mathbf{f})$ is slower than conjugate gradient method $(\mathrm{CG})$ for $l_{2}$-regularized $O(\mathbf{f})$. On a workstation with Intel Xeon X5550 CPU and 24G memory, it costs 23 seconds for NQP solver to restore an image with $1000 \times 1000$ pixels using Matlab while CG costs 1.9 seconds. The Matlab backslash operator costs much more time and memory. In the following evaluation, we use NQP solver for $l_{1}$-regularized $O(\mathbf{f})$.

\subsection{Effects of Restoration on Segmentation}

Data. Two sequences were captured at the resolution of $1040 * 1392$ pixels per image. Seq1: C2C12 muscle stem cells proliferated from $30+$ to $600+$ cells (imaged by ZEISS Axiovert $135 \mathrm{TV}$ phase contrast microscope at $5 \mathrm{X}$ magnification over 80 hours, 1000 images, Fig. [5(a,c)). Seq2: hundreds of bovine vascular cells migrated to the central image region (imaged by Leica DMI 6000B phase contrast microscope at 10X magnification over 16 hours, 200 images, Fig. $5(\mathrm{f}, \mathrm{h})$ ).

Metrics. We denote cell and background pixels as positive $(\mathrm{P})$ and negative (N) respectively. The true positive rate is defined as $\mathrm{TPR}=|\mathrm{TP}| /|P|$ where true positive (TP) stands for those cell pixels correctly labelled by both skilled human and our method. The false positive rate is defined as $\mathrm{FPR}=|\mathrm{FP}| /|N|$ where false positive (FP) are those cell pixels labelled by our method mistakenly. The accuracy is defined as $\mathrm{ACC}=(|\mathrm{TP}|+|N|-|\mathrm{FP}|) /(|P|+|N|)$.

Parameters. We normalized the restored images onto value range $[0,1]$. Based on a training pair of restored image and its ground truth mask (Seq1 uses the $500^{t h}$ image and Seq2 uses the $100^{t h}$ image), we applied a series of values between zero and one to threshold the restored image and compared with the ground truth to compute TPR and FPR scores, which provided a ROC curve. 

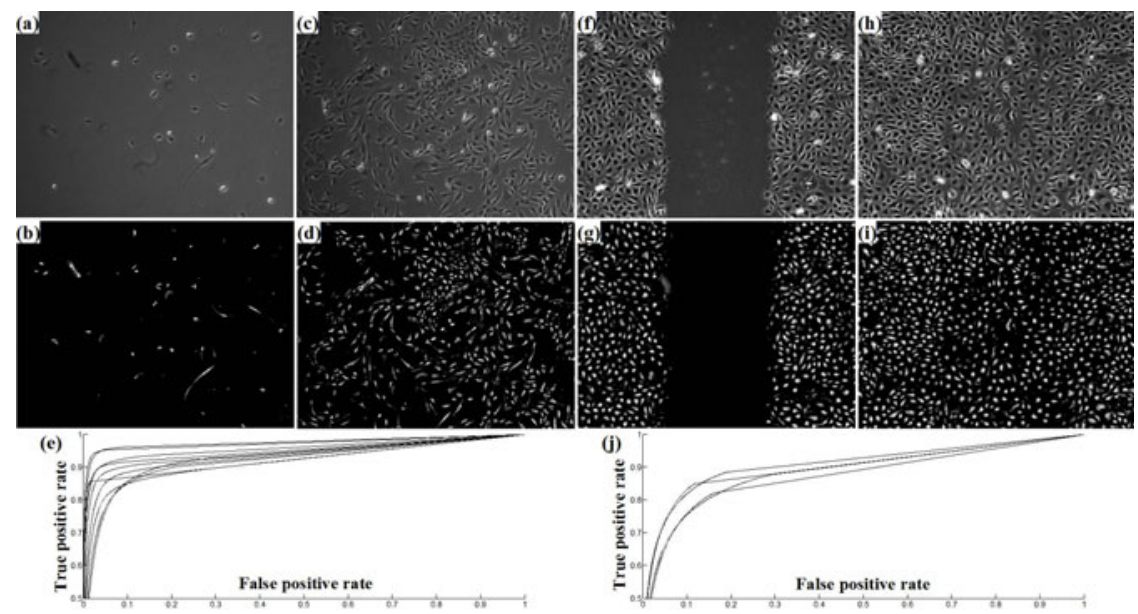

Fig. 5. Quantitative evaluation. (a-b, c-d, f-g, h-i): the pair of observed and restored images; (e) and (j): ROC curves for sequence 1 and 2 respectively.

Different parameter sets in the objective function generate different ROC curves. We searched the optimal parameter set $\left(\omega_{s}=1\right.$ and $\omega_{r}=.001$ in the evaluation) that has the largest area under the ROC curve (AUC). For the curve with the largest AUC, we searched the threshold that has the highest ACC to segment the restored image into a binary mask (both Seq1 and Seq2 got the best threshold equal to 0.22). We applied the learned parameters to all other images.

Evaluation. We manually labelled every $100^{\text {th }}$ image in Seq1 (2369 annotated cells, $8.6 \times 10^{5}$ cell pixels) and every $50^{\text {th }}$ image in Seq2 (2918 annotated cells, $1.1 \times 10^{6}$ cell pixels). It took human experts about 2 hours to label one thousand cell boundaries in an image. Fig. 5 shows some input and restored images with all ROC curves shown in Fig. [5(e) and (j), where ROC curves deviate gradually from the perfect top-left corner $(\mathrm{AUC}=1)$ as cell density increases. The average AUC is $94.2 \%$ (Seq1) and $89.2 \%$ (Seq2), and the average segmentation accuracy is $97.1 \%$ (Seq1) and $90.7 \%$ (Seq2).

\section{Conclusion}

We derived a linear imaging model representing the optical properties of phase contrast microscopes. Using our model, authentic artifact-free phase contrast images are restored by solving a constrained quadratic optimization problem. This work suggests that a better understanding of the optics of microscopes leads to better microscopy image analysis and interpretation. In particular, we have demonstrated that this approach can greatly faciliate the effort on microscopy image segmentation. 


\section{References}

1. Born, M., Wolf, E.: Principles of Optics, 6th edn. Pergamon Press, Oxford (1980)

2. Candes, E., Wakin, M., Boyd, S.: Enhancing Sparsity by Reweighted 11 Minimization. The Journal of Fourier Analysis and Applications 14(5), 877-905 (2008)

3. Gaskill, J.: Linear Systems, Fourier Transforms, and Optics. Wiley, Chichester (1978)

4. Grady, L.: Random Walks for Image Segmentation. IEEE Trans. on Pattern Analysis and Machine Intelligence 28(11), 1768-1783 (2006)

5. House, D., et al.: Tracking of Cell Populations to Understand their Spatio-Temporal Behavior in Response to Physical stimuli. In: MMBIA Workshop (2009)

6. Kim, S., et al.: An Interior-Point Method for Large-Scale 11-Regularized Least Squares. IEEE Journal of Selected Topics in Signal Processing 1(4) (2007)

7. Levin, A., et al.: A Closed Form Solution to Natural Image Matting. IEEE Trans. on Pattern Analysis and Machine Intelligence 30(2), 228-242 (2008)

8. Li, K., et al.: Cell Population Tracking and Lineage Construction with Spatiotemporal Context. Medical Image Analysis 12(5), 546-566 (2008)

9. Li, K., Kanade, T.: Nonnegative Mixed-Norm Preconditioning for Microscopy Image Segmentation. In: Prince, J.L., Pham, D.L., Myers, K.J. (eds.) IPMI 2009. LNCS, vol. 5636, Springer, Heidelberg (2009)

10. Murphy, D.: Fundamentals of Light Microscopy and Electronic Imaging. Wiley, Chichester (2001)

11. Sha, F., et al.: Multiplicative Updates for Nonnegative Quadratic Programming. Neural Computation 19(8), 2004-2031 (2007)

12. Smith, K., et al.: General Constraints for Batch Multiple-Target Tracking Applied to Large-Scale Videomicroscopy. In: Proceedings of CVPR (2008)

13. Wu, K., Gauthier, D., Levine, M.: Live Cell Image Segmentation. IEEE Trans. on Biomedical Engineering 42(1), 1-12 (1995)

14. Yang, F., et al.: Cell Segmentation, Tracking, and Mitosis Detection Using Temporal Context. In: Duncan, J.S., Gerig, G. (eds.) MICCAI 2005. LNCS, vol. 3749, pp. 302-309. Springer, Heidelberg (2005)

15. Zernike, F.: How I discovered phase contrast. Science 121, 345-349 (1955) 